cancer can reflect its dose-based advantages, associating with a remarkable reduction of patients' adverse reactions and a satisfactory therapeutic effect.

Disclosures None.

\section{HIGH EXPRESSION OF NANOG AND CRY1 IS INVOLVED WITH TUMOR PROGRESSION AND POOR PROGNOSIS IN PATIENTS WITH CERVICAL CANCER}

${ }^{1}$ Gwan Hee Han, ${ }^{2}$ Hanbyoul Cho. ${ }^{1}$ Department of Obstetrics and Gynecology, Yonsei University, College of Medicine; Department of Obstetrics and Gynecology, Kyung Hee University Hospital at Gangdong; ${ }^{2}$ Department of Obstetrics and Gynecology, Yonsei Univeristy, College of Medicine

\subsection{6/ijgc-2020-ESGO.14}

Introduction/Background Nanog is a well-known transcription factor regulating an embryonic stem cell maintenance. Recently, many evidences have been accumulated that overexpression of Nanog is intimately involved in tumorigenesis. However, the role of Nanog in cervical cancer has not been elucidated yet. Thus, we investigated the expression and clinical significance of Nanog in cervical cancer.

Methodology To evaluate the expression level of NANOG and CRY1, the immunohistochemistry on 170 cervical cancers and 263 cervical intraepithelial neoplasia (CIN) samples and the clinicopathologic variables of cervical cancer patients were compared to evaluate the significance of Nanog and CRY1 in cervical cancer. Also, in vitro assessment was performed by using Nanog knock down cervical cancer cell lines.

Results Nanog and CRY1 expression was higher in cervical cancer tissues than in CIN tissues and normal epithelial tissues (both $\mathrm{p}<0.001$ ). Importantly, Nanog and CRY1 overexpression was associated with poor chemoradiation response $(\mathrm{p}<$ $0.035, \mathrm{p}<0.003$, respectively). Multivariate survival analysis revealed that overexpression of Nanog (hazard ratio $=0.016$; $95 \%$ confidence interval [CI]: $1.25-9.27), \mathrm{p}=0.016)$ as an independent prognostic factor for overall survival. Also, the combination of high Nanog and CRY1 expression showed the highest hazard ratio (5.87; 95\% CI: 2.18-15.82, p < 0.001) for overall survival. In vitro results also demonstrated the knockdown of Nanog was associated with increased cell viability $(\mathrm{p}<0.001)$, migration $(\mathrm{p}<0.001)$ and growth $(\mathrm{p}<$ 0.001 ) supporting the oncogenic role of Nanog in cervical cancer.

Conclusion This study showed that overexpression of Nanog could be a good biomarker for the prediction of chemoradiation response. The results of survival analysis suggest a strong association between Nanog as well as CRY1 expression and poor overall survival, indicative of the potential role of this combination as a prognostic marker in clinical assessment.

Disclosures To the best of our knowledge, the named authors have no conflict of interest, financial or otherwise.

\section{PERFORMANCE AND DIAGNOSTIC ACCURACY OF HUMAN PAPILLOMAVIRUS TESTING ON URINE AND SELF-COLLECTED VAGINAL SAMPLES IN A REFERRAL POPULATION}

Hyun-Woong Cho, Jae Kwan Lee, Jin Hwa Hong. Korea University Guro Hospital
Introduction/Background Human papillomavirus (HPV) is well established as the main cause of cervical cancer. Non-invasive self-collected urine and vaginal sampling have the potential advantage of increasing patient compliance with cervical cancer screening.

Methodology Women referred for colposcopy at Korea University Guro Hospital, following abnormal cytology, were included this study. A total of 314 paired urine, vaginal and cervical samples were collected. Primary endpoints were sensitivity for CIN2+/CIN3 + and specificity for <CIN2; secondary endpoints were the relative accuracy of hrHPV test results in vaginal and urine samples versus cervical samples.

Results For clinician-collected cervical samples, Sejong Realtime HR-S HPV test sensitivity for detecting and specificity from were similar to well-established test (Anyplex ${ }^{\text {TM }}$ II HPV 28) [sensitivity for CIN3+ $(\mathrm{n}=109) \quad 93.27 \% \quad(95 \%$ confidence interval (CI), 86.62-97.25); CIN2+ $(n=130) \quad 92.74 \% \quad(95 \%$ CI, 86.67-96.63); specificity for $<$ CIN2 31.82\% (95\% CI, 25.01-39.25)]. All the paired differences (cervical versus urine sampling, cervix versus vaginal sampling) in sensitivity were statistically significant. However, among women with ASCUS/ LSIL cytology, hrHPV sensitivity on vaginal samples was comparable to that of cervical samples for detection of CIN2+ and CIN3 + lesions. In addition, hrHPV sensitivity of Anyplex II HPV 28 assay on urine was comparable to that of cervical samples for detection of CIN3 + lesions $(p=0.07)$ in women with ASCUS/LSIL cytology.

Conclusion HPV tests using urine and vaginal samples were still inferior to clinician-collected cervical samples in terms of detecting CIN2/3. However, these results indicate that combination of cytology with reflex hrHPV test using vaginal and urine samples may offer a reliable strategy for discriminating women at greater risk of precancerous lesion, increasing compliance of patients.

Disclosures I have no conflict of interest to disclose.

\section{SUCCOR CONE: IS IT CERVICAL CONIZATION A PROTECTIVE MANEUVER}

${ }^{1}$ Enrique Chacón, ${ }^{2}$ Nabil Manzour, ${ }^{3}$ José Ángel Mínguez, ${ }^{3}$ Daniel Vázquez, ${ }^{3}$ Teresa Castellano, ${ }^{3}$ Luisa Sánchez Lorenzo, ${ }^{3}$ Diego Salas, ${ }^{4} J u a n$ Luis Alcazar, ${ }^{3}$ Antonio Gonzalez-Martin, ${ }^{3}$ Luis M Chiva. ${ }^{1}$ Clínica Universidad de Navarra; Obstetrics and Gynecology; ${ }^{2}$ Clínica Universidad de Navarra; Clínica Universidad de Navarra; Gynecology; ${ }^{3}$ Clínica Universidad de Navarra; ${ }^{4}$ Clínica Universidad de Navarra; Clinica Universidad de Navarra

\subsection{6/ijgc-2020-ESGO.16}

Introduction/Background After the publication of the LACC trial, current evidence has focused on looking for the different reasons that have led to the open approach presenting better results than minimally invasive surgery (MIS). To date, no studies have considered the possible protective effect of cervical conization (CC).

Methodology Objective: The main goal of this study was to compare disease-free survival (DFS) and overall survival (OS) at 4.5 years in patients with stage IB1 cervical cancer who underwent radical hysterectomy (2013-2014) after CC vs nonCC patients. The secondary goal was to compare DFS by subgroups (tumor size and surgical approach in patients who underwent CC and those who did not) in the Propensity Matching Score (PMS) database.

Methods: Taking from 1272 patients from the European database belonging to the SUCCOR study and after applying 
the different inclusion and exclusion criteria, we obtained 1156 patients, 733 CC patients and 423 non-CC patients. Subsequently, and after analyzing the first results, we decided to homogenize our database by means of a PMS analysis, by this way, we obtained a new balanced population of 374 patients (187 CC patients and 187 non-CC patients).

Results In the general population, patients with CC present a $72 \%$ reduction in the risk of relapse compared to non-CC patients (HR: 0.28 95\% CI (0.17-0.46) p = 0.000) and a 90\% reduction in the risk of death (HR: 0.10 95\% CI $(0.03-$ 0.33) $\mathrm{p}=0.000)$, these differences may be due to the fact that both populations present differences.

After homogenizing our population using the PMS, we obtained that the reduction in the risk of relapse was $65 \%$ for patients who have CC (HR: 0.35 CI 95\% (0.16-0.75) p $=0.007)$ and $75 \%$ for the risk of death for the same cohort (HR: 0.25 95\% CI (0.07-0.90) p = 0.033).

Regarding the secondary objectives, we observed that the CC seems to have a protective effect in tumors between 2-4 cm (HR: 0.33 95\% CI $(0.11-0.99) \mathrm{p}=0.049)$. This same protective effect is observed in patients operated on by laparoscopy (HR: $0.3595 \%$ CI $(0.14-0.89) \mathrm{p}=0.028)$. Finally, the MIS patients who have CC do not present differences compared to those operated by the open approach, whether they are conized or non-conized (Log-Rank p $=0.439$ and LogRank p $=0.346)$.

Conclusion Patients undergoing CC have a significantly lower risk of relapse and death, this effect is more evident in those patients with $2-4 \mathrm{~cm}$ tumors or in those who are operated under MIS.

Disclosures I have nothing to disclose.

\section{DESIGN AND VALIDATION OF A RECURRENCE RISK PREDICTING SCORE IN EARLY STAGE CERVICAL CANCER AFTER RADICAL HYSTERECTOMY}

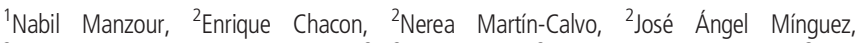
${ }^{2}$ Teresa Castellano, Daniel Vazquez ${ }^{2}$, ${ }^{2}$ Diego Salas, ${ }^{2}$ Antonio Gonzalez-Martin, ${ }^{3}$ Juan Luis Alcazar, '2Luis M Chiva. 'Clínica Universidad de Navarra; Clinica Universidad de Navarra; Gynecology; ${ }^{2}$ Clinica Universidad de Navarra; ${ }^{3}$ Clínica Universidad de Navarra; Clinica Universidad de Navarra
\end{abstract}

\subsection{6/ijgc-2020-ESGO.17}

Introduction/Background After the LACC trial, the scientific evidence has focused on confirming and finding the cause of why the open route presents better results than minimally invasive surgery (MIS). Even though the independent factors involved in relapse has not been studied.

\section{Methodology}

Primary objective to know the independent clinical, surgical and anatomopathological factors involved in the relapse of patients with stage IB1 cervical cancer who underwent radical hysterectomy (2013-2014).

Secondary objective To create a risk predictive index (RPI) that allows us to better select and stratify patients with a higher probability of relapse.

Methods Starting from 1272 patients from the European database belonging to the SUCCOR study and after applying the different inclusion and exclusion criteria we obtained 1156 patients. We randomly divided our sample into a test group and a validation group in a proportion of $60 \%$ to $40 \%$.
The test group was used to identify the variables independently associated with relapse and to define the relapse RPI. The RPI was applied to calculate a relapse risk score for each participant in the validation group. According to their risk of relapse, participants were classified into 3 risk groups.

Results Women who relapse are more likely to have tumours larger than $2 \mathrm{~cm}$ on imaging assessment (OR 2.15, 95\% CI 1.33- 3.5) and to undergo MIS (OR 1.61, 95\% CI 1.002.57). On the other hand, conisation is inversely associated with the risk of relapse (OR 0.31, 95\% CI 0.17- 0.60).

The AUC in the validation group for RPI is $(0.72 ; 95 \% \mathrm{CI}$ 0.65- 0.79).

Depending on their score, patients were classified at low, medium or high risk of relapse. The relapse rate observed in each group was $3.4 \%, 9.8 \%$ and $21.3 \%$ respectively.

With a median follow-up of 58 months, the mean DFS in the validation group for low, medium and high risk categories were 75.4 (95\% CI 73.8- 76.9), 75.5 (95\% CI 72.4- 78.5) and 64.1 (95\% CI 59.4- 68.9) months respectively $(\mathrm{P}<$ $0.001)$.

Conclusion Our risk predictor index proved to be valid and therefore may help to identify those patients who would benefit from adjuvant therapy and close follow-up after radical hysterectomy.

\section{SURVIVAL OUTCOMES OF PATIENTS WITH CLEAR CELL CARCINOMA CERVIX: A SINGLE INSTITUTIONAL RETROSPECTIVE ANALYSIS}

${ }^{1}$ Akhilesh CS Sudhakaran, ${ }^{2}$ Aswin Kumar, ${ }^{3}$ Francis James, ${ }^{2}$ Susan Mathews, ${ }^{2}$ John Joseph. ${ }^{1}$ Regional Cancer Centre, Trivandrum, Kerala, India; Radiation Oncology; ${ }^{2}$ Regional Cancer Centre, Trivandrum, Kerala, India; Division OF Gynec Oncology; ${ }^{3}$ Regional Cancer Centre; Regional Cencer Centre, Trivandrum, Kerala, India; Division OF Gynec Oncology

\subsection{6/ijgc-2020-ESG0.18}

Introduction/Background Cervical cancer is the most prevalent cancer and the fifth most common cause of cancer death in Indian women. Clear cell carcinoma of the cervix (CCCC) is rare, accounting for only $4 \%$ of all adenocarcinomas. CCCC can occur sporadically or in association with in-utero diethylstilbestrol (DES) exposure. There are no clear-cut treatment recommendations for the management of CCCC. Early-stage disease is usually treated by surgery and more locally advanced stages by chemoradiation followed by brachytherapy. Methodology

Aim: This study aimed to assess the survival outcomes and patterns of failure of patients with CCCC.

Settings and Design: Retrospective study done at Regional Cancer Centre, Thiruvananthapuram, Kerala, India

Material and Methods: Case records of all the patients with CCCC who were diagnosed and treated between 1995 and 2015 were reviewed for clinical, pathological and treatment characteristics.

Statistical analysis: Disease-free survival (DFS) and overall survival (OS) were estimated using the Kaplan-Meier method.

Results The diagnosis of clear cell carcinoma of the cervix was confirmed in 15 patients. The median age was 53 years. $20 \%$ of the patients were in the International Federation of Gynaecology and Obstetrics (FIGO) stage I, 60\% in stage II, $7 \%$ in Stage III and $13 \%$ in stage IVA. Stage IB and IVA patients were managed surgically, and adjuvant therapy decided based on the tumour pathology. Stage IIB and IIIB 